\title{
Impact of Cannabis Use on Long-Term Remission in Bipolar I and Schizoaffective Disorder
}

\author{
Sung-Wan Kim ${ }^{1}$, Seetal Dodd ${ }^{2,3}$, Lesley Berk ${ }^{2,3,4}$, Jayashri Kulkarni ${ }^{5}$, Anthony de Castella ${ }^{5}$, \\ Paul B. Fitzgerald ${ }^{5}$, Jae-Min Kim¹, Jin-Sang Yoon', and Michael Berk ${ }^{2,3,6,7} \bowtie$ \\ 1'Department of Psychiatry, Chonnam National University Medical School, Gwangju, Republic of Korea \\ ${ }^{2}$ IMPACT Strategic Research Centre, School of Medicine, Deakin University, Geelong, Australia \\ ${ }^{3}$ Department of Psychiatry, University of Melbourne, Melbourne, Australia \\ ${ }^{4}$ Centre for Mental Health and Wellbeing Research, School of Psychology, Faculty of Health Deakin University, Geelong, Australia \\ ${ }^{5}$ Monash Alfred Psychiatry Research Centre, The Alfred Hospital and Monash University, Melbourne, Australia \\ ${ }^{6}$ Orygen Youth Health Research Centre, Melbourne, Australia \\ ${ }^{7}$ Florey Institute for Neuroscience and Mental Health, Melbourne, Australia
}

Objective To investigate the impact of regular cannabis use on long-term remission of mood symptoms in bipolar spectrum disorders. Methods The 24-month prospective observational study included patients $(\mathrm{n}=239)$ with bipolar I disorder and schizoaffective disorder, bipolar type. Participants were classified as regular cannabis users (three times or more per week) or non-users. The primary outcome measure was the achievement of remission on the evaluations during the 24 months.

Results Of the 234 participants for whom data was available, 25 (10.7\%) were regular cannabis users, and the group comprised significantly more males than females. In the total population, cannabis use was significantly associated with decreased likelihood of remission during the 24-month follow-up period. Subgroup analyses showed that cannabis use was significantly associated with lower remission rates on the Hamilton Depression Rating Scale in females $(n=139)$ and patients prescribed mood stabilizers alone $(n=151)$, whereas in males $(n=95)$ and patients prescribed olanzapine and/or a mood stabilizer $(n=83)$, cannabis use was significantly associated with lower remission rates on the Young Mania Rating Scale. Remission rates were lowest in the concurrent cannabis and tobacco smoking group $(n=22)$ followed by the tobacco smoking only group $(n=97)$, and the non-smoker group $(n=116)$. The post-hoc analysis revealed that all remission rates were significantly lower in the concurrent cannabis and the tobacco smoking group compared to the non-smoker group.

Conclusion Cannabis use negatively affects the long-term clinical outcome in patients with bipolar spectrum disorders. A comprehensive assessment and integrated management of cannabis use are required to achieve better treatment outcomes for bipolar spectrum disorders.

Psychiatry Investig 2015;12(3):349-355

Key Words Cannabis, Bipolar disorder, Schizoaffective disorder, Observational study, Substance, Remission.

\section{INTRODUCTION}

Cannabis use is common in psychiatric populations; ${ }^{1,2}$ it is a well-documented risk factor for schizophrenia and is associated with a poor prognosis for the condition. ${ }^{3-6}$ Cannabis is one of the most commonly abused drugs among patients with

Received: April 26, 2014 Revised: August 25, 2014

Accepted: August 29, 2014 Available online: July 6, 2015

$\triangle$ Correspondence: Michael Berk, MD, PhD

School of Medicine, Deakin University, 1 Gheringhap St., Geelong, VIC 3220, Australia

Tel: +61352267450 , Fax: +61352267436

E-mail: MIKEBE@BarwonHealth.org.au

(a) This is an Open Access article distributed under the terms of the Creative Commons Attribution Non-Commercial License (http://creativecommons.org/licenses/bync/3.0) which permits unrestricted non-commercial use, distribution, and reproduction in any medium, provided the original work is properly cited. bipolar disorder. ${ }^{1,7,8}$ Several cross-sectional studies of patients with bipolar disorder have shown that cannabis use is associated with an earlier age of onset, severe mood symptoms, greater disability, and a higher suicide risk compared with non-users. ${ }^{2,69-12} \mathrm{~A}$ few prospective studies have revealed that cannabis users exhibit poor adherence to treatment and have higher levels of overall illness severity, mania, and psychosis than do non-users. ${ }^{13-15}$ However, the effect of cannabis on the long-term clinical course of patients with bipolar spectrum disorders have been relatively less studied, whereas several prospective studies have examined the effects of alcohol use disorders and tobacco smoking on the course of bipolar illness. ${ }^{16-18}$

Previous studies of bipolar disorder have found that cannabis use is more frequent in males than in females. ${ }^{8,19,20}$ More- 
over, gender differences in illness characteristics and course have been widely documented in psychiatric illnesses including bipolar disorder. ${ }^{2,20-22}$ However, to our knowledge, no study of the effect of cannabis use on long-term clinical outcomes has investigated gender differences.

Our 24-month prospective study investigated the impact of cannabis use on the clinical course of patients with bipolar spectrum disorders with a focus on long-term remission of mood symptoms according to gender and type of medication. The present study analyzed data from the Bipolar Comprehensive Outcomes Study (BCOS), ${ }^{23}$ a 2 -year, prospective, naturalistic observational study designed to improve understanding of the treatment and outcomes of patients diagnosed with bipolar I disorder or schizoaffective disorder.

\section{METHODS}

\section{Study design and participants}

The rationale and design of the BCOS have been detailed previously. ${ }^{23,24}$ Briefly, participants were enrolled in the study if they met the diagnostic criteria for bipolar I disorder or schizoaffective disorder, bipolar type, were aged 18 years or older, and were willing and able to comply with the study requirements. To obtain a representative sample of participants, the exclusion criteria were minimal. Participants could commence participation in the study at any phase of their illness. As this was an observational study, participants were not randomised to treatment groups but instead received their usual care, which could be varied by their treating clinician. At baseline, participants had to be receiving at least one of several conventional mood stabilizers (lithium carbonate, sodium valproate, or carbamazepine), olanzapine as a mood stabilizer, or a combination of a conventional mood stabilizer plus olanzapine. All treatment decisions were made by the participant's primary treating clinicians, independent of the study. Participants were recruited from October 2003 to May 2008 from Australian public hospitals and private clinics. Trained evaluators assessed participants on nine separate occasions: at study entry and every 3 months up to 24 months. All participants provided informed consent to participate in the study, which was conducted in accordance with Australian ethics and the Helsinki Declaration of 1975 and approved by the relevant ethics committees.

\section{Procedures and measures}

Diagnoses were confirmed by the Mini-International Neuropsychiatric Interview (MINI) version 5, a semi-structured interview designed to identify major Axis I psychiatric disorders including alcohol dependence and abuse. ${ }^{25}$ Suicide risk in the past month was also measured by six questions during the MINI interview. High suicide risk was defined by scores on the relevant questions on the MINI interview.

The primary outcome measure of this study was the rate of achieving remission over the nine evaluation points (number of remissions/9) during the 24 months. Missing data resulting from non-visits were operationally defined as indicative of non-remission. Symptomatic remission was defined as a Young Mania Rating Scale (YMRS) ${ }^{26}$ total score of $\leq 12^{27}$ or a 21-item Hamilton Depression Rating Scale (HAMD-21) ${ }^{28}$ total score of $\leq 8 .^{29,30}$ Total remission was defined as remission of both YMRS and HAMD-21 scores.

Our key independent variable was cannabis use. Participants were questioned about cannabis use at visit 1 using the Habits form. ${ }^{31}$ Frequency of cannabis use during the past 3 months was investigated. Participants who reported regular cannabis use 3 or more days per week were classified as cannabis users, which was consistent with previous studies. ${ }^{32,33}$ The remaining participants were classified as cannabis nonusers. In terms of tobacco smoking, the BCOS study classified participants as those who smoked tobacco daily and those who did not.

\section{Statistical analysis}

Sociodemographic and clinical variables of the cannabis user and non-user groups were compared using independent chi-squared tests, t-tests, or Mann-Whitney U-tests as appropriate. The effect of cannabis use on total, 21-item Hamilton Depression Rating Scale (HAMD-21), and the Young Mania Rating Scale (YMRS) remission rates during the 24-month follow-up period were compared using the Mann-Whitney U-test. Additionally, comparisons were made across gender and medication type. Spearman's correlation test was used to assess the relationship between age and remission rates. In order to explore the effect of tobacco smoking on remission rate, the participants were divided into three subgroups: concurrent cannabis and tobacco smoking, tobacco smoking only, and non-smoking. Remission rates among the three groups were compared using the Kruskal-Wallis test and post hoc paired comparisons were conducted using the MannWhitney U-test with the Bonferroni correction. Statistical significance was defined as $p$-values of $\leq 0.05$. All statistical tests were two tailed.

\section{RESULTS}

A total of 239 participants were enrolled in the BCOS study. We were unable to obtain information on cannabis use from 5 of the 239 participants. Of the 234 participants whose data were analyzed, 213 (91.0\%) completed the 24-month study. The mean age of participants was $41.8 \pm 12.7$ years and 
$59.4 \%$ were female. A total of 172 participants were diagnosed with bipolar I disorder and 62 were diagnosed with schizoaffective disorder, bipolar type.

Sociodemographic and clinical characteristics were compared according to cannabis use (Table 1). A total of 25 (10.7\%) subjects regularly used cannabis. The mean age of cannabis users was significantly lower than that of non-users. Significantly more males than females were cannabis users. The percentage of daily tobacco smokers was significantly higher among cannabis users than among non-users; only 3 (12.0\%) regular cannabis users did not smoke daily. Baseline YMRS scores were significantly higher in cannabis users than in non-users. Baseline HAMD-21 scores tended to be higher among cannabis users than non-users; however, the difference was not statistically significant $(\mathrm{p}=0.051)$. There was a nonsignificant trend for cannabis users to be at higher risk of suicide than non-users at baseline $(\mathrm{p}=0.069)$. No significant difference of concurrent alcohol dependence and alcohol abuse existed between cannabis users and non-users.

The Spearman's correlation coefficients revealed no significant difference between age and total ( $\mathrm{rho}=0.066 ; \mathrm{p}=0.312$ ), HAMD-21 (rho=0.065; $\mathrm{p}=0.314$ ), or YMRS ( $\mathrm{rho}=0.102 ; \mathrm{p}=$ 0.114 ) remission rates. Table 2 shows remission status among cannabis users and non-users according to gender and medication type. Cannabis use was significantly associated with low total remission and HAMD-21 remission rates during the 24-month follow-up period. YMRS remission rates were lower in cannabis users than in non-users; however, the differ-

Table 1. Sociodemographic and clinical characteristics according to cannabis use

\begin{tabular}{|c|c|c|c|c|}
\hline & $\begin{array}{c}\text { Total } \\
234(100 \%)\end{array}$ & $\begin{array}{c}\text { Cannabis user } \\
25(10.7 \%)\end{array}$ & $\begin{array}{c}\text { Non-user } \\
209(89.3 \%)\end{array}$ & p-value \\
\hline Age, years (SD) & $41.8(12.7)$ & $32.3(7.6)$ & $43.0(12.8)$ & $<0.001^{*}$ \\
\hline Age at onset, years (SD) & $22.9(9.6)$ & $22.1(6.7)$ & $22.9(9.9)$ & 0.694 \\
\hline Gender; male & $95(40.6 \%)$ & $16(64.0 \%)$ & $79(37.8 \%)$ & $0.012^{*}$ \\
\hline Diagnosis, bipolar disorder & $172(73.5 \%)$ & $18(72.0 \%)$ & $154(73.7 \%)$ & 0.857 \\
\hline Current psychotic feature & $50(21.4 \%)$ & $6(24.0 \%)$ & $44(21.1 \%)$ & 0.734 \\
\hline Medication; Olanzapine & $83(35.5 \%)$ & $7(28.0 \%)$ & $76(36.4 \%)$ & 0.409 \\
\hline Hospitalization in 3 month & $77(32.9 \%)$ & $7(28.0 \%)$ & $70(33.5 \%)$ & 0.581 \\
\hline High suicide risk & $51(21.8 \%)$ & $9(36.0 \%)$ & $42(20.1 \%)$ & 0.069 \\
\hline Daily smoker & $119(50.9 \%)$ & $22(88.0 \%)$ & $97(46.4 \%)$ & $<0.001^{*}$ \\
\hline Alcohol dependence & $40(17.1 \%)$ & $4(16.0 \%)$ & $36(17.2 \%)$ & 1.000 \\
\hline Alcohol abuse & $26(11.1 \%)$ & $4(16.0 \%)$ & $22(10.5 \%)$ & 0.496 \\
\hline Baseline HAMD-21, Median (IQR) & $13(7-19)$ & $16(10-25)$ & $13(7-18)$ & 0.051 \\
\hline Baseline YMRS, Median (IQR) & $5(2-13)$ & $14(4-24)$ & $5(2-11)$ & $0.001^{*}$ \\
\hline
\end{tabular}

${ }^{*} \mathrm{p}<0.05$. SD: standard deviation, HAMD-21: 21-item Hamilton Depression Rating Scale, YMRS: Young Mania Rating Scale, IQR: interquartile range

Table 2. Remission rates among cannabis users and non-users according to gender and medication type during the 24-month follow-up period

\begin{tabular}{|c|c|c|c|c|c|c|c|c|c|c|}
\hline & \multirow[b]{2}{*}{$\mathrm{N}$} & \multicolumn{3}{|c|}{ Total remission } & \multicolumn{3}{|c|}{ HAMD-21 remission } & \multicolumn{3}{|c|}{ YMRS remission } \\
\hline & & $\begin{array}{c}\text { Cannabis } \\
\text { users }\end{array}$ & Non-users & $\mathrm{p}$-value & $\begin{array}{c}\text { Cannabis } \\
\text { users }\end{array}$ & Non-users & $\mathrm{p}$-value & $\begin{array}{c}\text { Cannabis } \\
\text { users }\end{array}$ & Non-users & p-value \\
\hline Total & 234 & $11(0-39)$ & $33(11-67)$ & $0.025^{*}$ & $22(0-44)$ & $33(11-67)$ & $0.035^{*}$ & 78 (44-94) & $89(67-100)$ & 0.051 \\
\hline \multicolumn{11}{|l|}{ Gender } \\
\hline Female & 139 & $0(0-17)$ & $33(11-67)$ & $0.005^{*}$ & $0(0-17)$ & $33(11-67)$ & $0.002^{*}$ & $89(39-100)$ & $89(67-100)$ & 0.802 \\
\hline Male & 95 & $28(3-53)$ & $33(0-67)$ & 0.449 & $39(11-61)$ & $33(0-67)$ & 0.601 & $78(44-89)$ & $89(67-100)$ & $0.019^{*}$ \\
\hline \multicolumn{11}{|l|}{ Medication } \\
\hline Mood stabilizer alone & 151 & $11(0-33)$ & $33(11-67)$ & $0.028^{*}$ & $17(0-44)$ & $33(11-67)$ & $0.050^{*}$ & $83(44-100)$ & $89(67-100)$ & 0.374 \\
\hline $\begin{array}{l}\text { Olanzapine } \pm \text { mood } \\
\text { stabilizer }\end{array}$ & 83 & $22(0-44)$ & $33(0-67)$ & 0.475 & $22(0-44)$ & $33(11-67)$ & 0.380 & $78(0-78)$ & $89(78-100)$ & $0.030^{*}$ \\
\hline
\end{tabular}

Values indicate median and interquartile range. ${ }^{*} \mathrm{p}<0.05$. HAMD-21: 21 -item Hamilton Depression Rating Scale, YMRS: Young Mania Rating scale 
ence did not reach statistical significance $(\mathrm{p}=0.051)$.

The analysis of remission rates in cannabis users and nonusers across gender and medication type revealed that cannabis use was significantly associated with lower total remission and HAMD-21 remission rates in females $(n=139)$, whereas it was significantly associated with lower YMRS, but not HAMD-21, remission rates in males $(n=95)$. Cannabis use was significantly associated with lower total remission and HAMD-21 remission rates among patients who were prescribed mood stabilizers alone $(n=151)$; however, cannabis use was significantly associated with lower YMRS remission rates in patients who took olanzapine and/or mood stabilizers $(n=83)$.

Figure 1 shows total, HAMD-21, and YMRS remission rates among the concurrent cannabis and tobacco smoking group ( $n=22)$, tobacco smoking only $(n=97)$, and non-smoker groups $(n=116)$ during the 24 -month follow-up period. All remission rates (total, HAMD-21, and YMRS) were significantly different among the three groups $(\mathrm{p}=0.011,0.016$, and 0.012 , respectively). The remission rates were lowest in the concurrent cannabis and tobacco smoking group and the non-smoker group had the highest remission rates. The post hoc analysis revealed that all remission rates (total, HAMD21, and YMRS) were significantly lower in the concurrent cannabis and tobacco-smoking group compared to the nonsmoker group ( $p=0.008,0.012$, and 0.009 , respectively). The remission rates in the tobacco smoking only group were higher than in the non-smoker group ( $\mathrm{p}=0.048-0.050)$, but the difference did not reach statistical significance after the Bonferroni correction ( $\mathrm{p}=0.0169$ ). The remission rates were not significantly different between the tobacco only and concurrent cannabis and tobacco groups (all p>0.08).

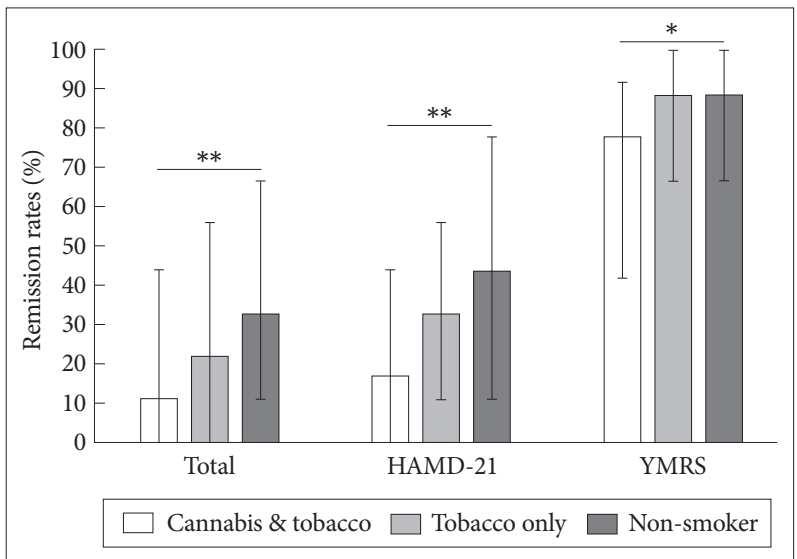

Figure 1. Comparison of remission rates according to cannabis and tobacco use. Bar indicates median and interquartile range. ${ }^{*} \mathrm{p}$ value $<0.05,{ }^{* *}$ p-value $<0.01$ (statistically significant after the Bonferroni correction). HAMD-21: 21-item Hamilton Depression Rating Scale, YMRS: Young Mania Rating scale.

\section{DISCUSSION}

Our findings show that cannabis use is associated with decreased likelihood of long-term remission in bipolar spectrum disorders. In particular, we observed interaction effects of cannabis use and mood symptoms on gender and medication type. That is, cannabis use was associated with lower remission rates for depressive symptoms in females and for manic symptoms in males. Moreover, remission rates for depressive symptoms were lower in cannabis users prescribed mood stabilizers alone, whereas remission rates for manic symptoms were lower in cannabis users prescribed olanzapine. To our knowledge, this study is the first to demonstrate differing effects of cannabis use on polarity specific long-term remission rates (24-month period) according to gender and type of medication in patients with bipolar spectrum disorders.

A few studies have demonstrated an association between cannabis use and severe manic symptoms, but not depression, in patients with bipolar disorder. ${ }^{13,14}$ Furthermore, cannabis use has been shown to increase the risk of manic symptoms in the general population. ${ }^{34}$ However, a recent study showed that the association between cannabis use and mood symptoms is complex and more strongly correlated with depression than mania. ${ }^{35}$ Our results revealed interactions of depressive and manic symptom remission rates on gender and type of medication.

Our finding of an association between cannabis use and lower remission rates for depressive symptoms in females supports a previous study showing that cannabis use in teenage girls, but not boys, predicted high rates of depression. ${ }^{36}$ Moreover, cumulative evidence suggests that cannabis use increases the risk for unipolar depression. ${ }^{37,38}$ This association may be linked to the greater vulnerability of women to developing depression. ${ }^{36,39}$ Childhood sexual abuse, which is widespread among female cannabis users with bipolar disorder, ${ }^{12}$ is associated with poor outcomes for depression and should be considered a contributing factor to the effect of cannabis use on clinical outcome. ${ }^{40,41}$ Similarly, abuse is linked to the development of maladaptive cognitive schemas and extreme attributions, which too are risks for poorer outcomes. ${ }^{42,43}$

Cannabis may diminish the effect of medications used to treat bipolar spectrum disorders. Our results showed that olanzapine was associated with lower remission rates for manic symptoms in patients with bipolar spectrum disorder who were cannabis users. Investigations into the effect of cannabis on the inhibition and induction of human liver cytochrome P450 (CYP450) isoforms generally reflect a low risk of clinically significant drug interactions with most use, although in vivo studies in humans have not been conducted. ${ }^{44,45}$ However, smoked cannabis and tobacco induce hepatic 
CYP 1A2 enzyme activity; thus, the systemic effects of CYP 1A2 substrates, such as olanzapine, may be decreased in individuals who smoke cannabis. ${ }^{44-47}$ Furthermore, poor adherence to medications, which is common among cannabis users in bipolar populations, may contribute to unfavorable outcomes. ${ }^{15,48-50}$

A high rate of co-use of cannabis with tobacco smoking and alcohol use has been observed in patients with bipolar and other psychiatric disorders. ${ }^{2,15,16,32,51,52}$ A recent study found that co-occurring cannabis use disorder and nicotine dependence was associated with a higher prevalence of bipolar disorder compared with cannabis use disorder alone..$^{53}$ In our study, alcohol dependence and abuse were not associated with cannabis use. However, cannabis use was highly associated with tobacco smoking. Previous studies, including the BCOS, have found that tobacco smoking is associated with poor mental health outcomes among patients with bipolar spectrum disorder. ${ }^{17,18,54}$ Our results revealed a tendency for lower remission rates in tobacco only smokers compared with non-smokers. Furthermore, our findings suggest that concurrent cannabis and tobacco smoking has a cumulative effect on unfavorable outcomes in patients with bipolar spectrum disorders. Ideally, a comparison of cannabis users who did and did not smoke tobacco would help differentiate the effects of tobacco and cannabis smoking on remission rates. However, the number of cannabis users in our study population who did not smoke tobacco $(n=3)$ was too small to perform a statistical analysis. Further studies that include subjects who smoke cannabis but not tobacco are warranted.

Substance use disorders including cannabis use often go unrecognized or are viewed as a secondary problem in psychiatric treatment settings. ${ }^{55}$ However, our results, showing a negative impact of cannabis use on the clinical course of patients with bipolar spectrum disorders, highlight the need to actively assess and manage cannabis use problems. A comprehensive evaluation of patients with bipolar disorders (particularly males) should include a systematic assessment of substance use problems including cannabis use. ${ }^{2}$ Furthermore, specialized treatment approaches should be available for this vulnerable population including motivational interview and cognitive behavioral therapy for co-occurring bipolar and cannabis use. ${ }^{55}$ Further study is required to investigate the impact of effective management of cannabis use on the clinical course of patients with bipolar spectrum disorders. In addition, the biological substrates associated with comorbid cannabis use in bipolar spectrum disorders should be investigated to clarify the mechanisms underlying the negative impact and to develop effective and safe treatment strategies.

Our study has several limitations that should be considered when interpreting our results. First, cannabis use was investi- gated only cross-sectionally and there was no longitudinal evaluation. Regular checks on cannabis use in patients with bipolar spectrum disorders would provide more information about longitudinal outcomes. In addition, the investigation of the relative sequence of cannabis use onset and bipolar disorder onset would provide a deeper understanding of the complex associations between these two conditions. Second, the potential impact of concomitant psychosocial therapies on the outcomes was not evaluated in this study. Third, HAMD21 and YMRS scores were used to evaluate symptoms in the week prior to the assessment visit. Given the fluctuating course of symptoms, this timing may not adequately reflect the symptoms across the 3-month interval between visits. However, the 2-year prospective observation period provided an extended period of observation that enabled examination of the fluctuating course of illnesses and outcomes. Finally, our sample and setting might have resulted in selection bias and unmeasured confounding variables. In addition, the quality of treatment was not controlled strictly in this study. However, our prospective, pragmatic study design, high 2-year retention rate, and inclusion of patients at all levels of symptom severity and treatment allows for broad generalizability. ${ }^{30}$

In conclusion, regular cannabis use has a negative effect on the clinical course of bipolar spectrum disorders. Individuals with bipolar spectrum disorders who are regular cannabis users are a vulnerable population. ${ }^{9}$ Given the clinical significance of cannabis use in bipolar spectrum disorders, routine comprehensive and regular assessments of cannabis use are recommended. Furthermore, special attention to, and management of, patients with these substance problems must be integrated into the treatment strategy to achieve better therapeutic outcomes for bipolar spectrum disorders.

\section{Acknowledgments}

The Bipolar Comprehensive Outcomes Study (BCOS) was funded by an unconditional research grant provided by Eli Lilly Australia. Lilly had no role in the collection, analysis, interpretation of data, the writing of the report, and in the decision to submit the paper for publication.

SWK is supported by Research Institute of Medical Sciences, Chonnam National University, Korea. LB is supported by an Alfred Deakin Postdoctoral Research Fellowship. PBF is supported by an NHMRC Practitioner Fellowship (606907). MB is supported by a NHMRC Senior Principal Research Fellowship (1059660).

\section{REFERENCES}

1. Saddichha S, Sur S, Sinha BN, Khess CR. How is substance use linked to psychosis? A study of the course and patterns of substance dependence in psychosis. Subst Abus 2010;31:58-67.

2. Lev-Ran S, Le Foll B, McKenzie K, George TP, Rehm J. Bipolar disorder and co-occurring cannabis use disorders: characteristics, co-morbidities and clinical correlates. Psychiatry Res 2013;209:459-465.

3. Linszen DH, Dingemans PM, Lenoir ME. Cannabis abuse and the course of recent-onset schizophrenic disorders. Arch Gen Psychiatry 1994;51:273-279. 
4. Caspari D. Cannabis and schizophrenia: results of a follow-up study. Eur Arch Psychiatry Clin Neurosci 1999;249:45-49.

5. Grech A, Van Os J, Jones PB, Lewis SW, Murray RM. Cannabis use and outcome of recent onset psychosis. Eur Psychiatry 2005;20:349-353.

6. De Hert M, Wampers M, Jendricko T, Franic T, Vidovic D, De Vriendt $\mathrm{N}$, et al. Effects of cannabis use on age at onset in schizophrenia and bipolar disorder. Schizophr Res 2011;126:270-276.

7. Estroff TW, Dackis CA, Gold MS, Pottash AL. Drug abuse and bipolar disorders. Intl J Psychiatry Med 1985;15:37-40.

8. Cassidy F, Ahearn EP, Carroll BJ. Substance abuse in bipolar disorder. Bipolar Disord 2001;3:181-188.

9. Agrawal A, Nurnberger JI Jr, Lynskey MT; Bipolar Genome Study. Cannabis involvement in individuals with bipolar disorder. Psychiatry Res 2011;185:459-461.

10. Lagerberg TV, Sundet K, Aminoff SR, Berg AO, Ringen PA, Andreassen OA, et al. Excessive cannabis use is associated with earlier age at onset in bipolar disorder. Eur Arch Psychiatry Clin Neurosci 2011;261: 397-405.

11. Lagerberg TV, Kvitland LR, Aminoff SR, Aas M, Ringen PA, Andreassen OA, et al. Indications of a dose-response relationship between cannabis use and age at onset in bipolar disorder. Psychiatry Res 2014;215: 101-104.

12. Aas M, Etain B, Bellivier F, Henry C, Lagerberg T, Ringen A, et al. Additive effects of childhood abuse and cannabis abuse on clinical expressions of bipolar disorders. Psychol Med 2014;44:1653-1662.

13. Strakowski SM, DelBello MP, Fleck DE, Arndt S. The impact of substance abuse on the course of bipolar disorder. Biol Psychiatry 2000;48: 477-485.

14. Baethge C, Baldessarini RJ, Khalsa HM, Hennen J, Salvatore P, Tohen M. Substance abuse in first-episode bipolar I disorder: indications for early intervention. Am J Psychiatry 2005;162:1008-1010.

15. van Rossum I, Boomsma M, Tenback D, Reed C, van Os J; EMBLEM Advisory Board. Does cannabis use affect treatment outcome in bipolar disorder? A longitudinal analysis. J Nerv Ment Dis 2009;197:35-40.

16. Strakowski SM, DelBello MP, Fleck DE, Adler CM, Anthenelli RM, Keck PE Jr, et al. Effects of co-occurring alcohol abuse on the course of bipolar disorder following a first hospitalization for mania. Arch Gen Psychiatry 2005;62:851-858.

17. Berk M, Ng F, Wang WV, Tohen M, Lubman DI, Vieta E, et al. Going up in smoke: tobacco smoking is associated with worse treatment outcomes in mania. J Affect Disord 2008;110:126-134.

18. Dodd S, Brnabic AJ, Berk L, Fitzgerald PB, de Castella AR, Filia S, et al. A prospective study of the impact of smoking on outcomes in bipolar and schizoaffective disorder. Compr Psychiatry 2010;51:504-509.

19. Bakare MO, Agomoh AO, Ebigbo PO, Onyeama GM, Eaton J, Onwukwe JU, et al. Co-morbid disorders and sexual risk behavior in Nigerian adolescents with bipolar disorder. Int Arch Med 2009;2:16.

20. Cotton SM, Lambert M, Berk M, Schimmelmann BG, Butselaar FJ, McGorry PD, et al. Gender differences in first episode psychotic mania. BMC Psychiatry 2013;13:82.

21. Farren CK, Snee L, McElroy S. Gender differences in outcome at 2-year follow-up of treated bipolar and depressed alcoholics. J Stud Alcohol Drugs 2011;72:872-880.

22. Nivoli AM, Pacchiarotti I, Rosa AR, Popovic D, Murru A, Valenti M, et al. Gender differences in a cohort study of 604 bipolar patients: the role of predominant polarity. J Affect Disord 2011;133:443-449.

23. Kulkarni J, Berk M, Fitzgerald PB, de Castella AR, Montgomery W, Kelin K, et al. The Bipolar Comprehensive Outcomes Study (BCOS): baseline findings of an Australian cohort study. J Affect Disord 2008;107: 135-144.

24. Berk M, Dodd S, Callaly P, Berk L, Fitzgerald P, de Castella AR, et al. History of illness prior to a diagnosis of bipolar disorder or schizoaffective disorder. J Affect Disord 2007;103:181-186.

25. Sheehan DV, Lecrubier Y, Sheehan KH, Amorim P, Janavs J, Weiller E, et al. The Mini-International Neuropsychiatric Interview (M.I.N.I.): the development and validation of a structured diagnostic psychiatric interview for DSM-IV and ICD-10. J Clin Psychiatry 1998;59(Suppl 20): 22-33.

26. Young RC, Biggs JT, Ziegler VE, Meyer DA. A rating scale for mania: reliability, validity and sensitivity. Br J Psychiatry 1978;133:429-435.

27. Lam RW, Michalak EE, Swinson RP. Assessment Scales in Depression, Mania and Anxiety. London and New York: Taylor and Francis; 2005.

28. Hamilton M. A rating scale for depression. J Neurol Neurosurg Psychiatry 1960;23:56-62.

29. Tohen M, Frank E, Bowden CL, Colom F, Ghaemi SN, Yatham LN, et al. The International Society for Bipolar Disorders (ISBD) Task Force report on the nomenclature of course and outcome in bipolar disorders. Bipolar Disord 2009;11:453-473.

30. Kulkarni J, Filia S, Berk L, Filia K, Dodd S, de Castella A, et al. Treatment and outcomes of an Australian cohort of outpatients with bipolar I or schizoaffective disorder over twenty-four months: implications for clinical practice. BMC Psychiatry 2012;12:228.

31. Guy W. ECDEU Assessment Manual for Psychopharmacology. Rockville, MD: U.S. National Institute of Health, Psychopharmacology Research Branch; 1976.

32. Heffner JL, DelBello MP, Fleck DE, Anthenelli RM, Strakowski SM. Cigarette smoking in the early course of bipolar disorder: association with ages-at-onset of alcohol and marijuana use. Bipolar Disord 2008; 10:838-845.

33. Macgregor S, Payne J. Cannabis Use and Mental Health: Findings from a Sample of Offenders in Police Custody. Criminal Justice Bulletin 9. Sydney: National Cannabis Prevention and Information Centre; 2012.

34. Henquet C, Krabbendam L, de Graaf R, ten Have M, van Os J. Cannabis use and expression of mania in the general population. J Affect Disord 2006;95:103-110.

35. Strakowski SM, DelBello MP, Fleck DE, Adler CM, Anthenelli RM, Keck PE Jr, et al. Effects of co-occurring cannabis use disorders on the course of bipolar disorder after a first hospitalization for mania. Arch Gen Psychiatry 2007;64:57-64.

36. Patton GC, Coffey C, Carlin JB, Degenhardt L, Lynskey M, Hall W. Cannabis use and mental health in young people: cohort study. BMJ 2002;325:1195-1198.

37. Rey JM, Tennant CC. Cannabis and mental health. BMJ 2002;325: 1183-1184.

38. Degenhardt L, Hall W, Lynskey M. Exploring the association between cannabis use and depression. Addiction 2003;98:1493-1504.

39. Fergusson DM, Horwood LJ. Early onset cannabis use and psychosocial adjustment in young adults. Addiction 1997;92:279-296.

40. Conus P, Cotton S, Schimmelmann BG, Berk M, Daglas R, McGorry $\mathrm{PD}$, et al. Pretreatment and outcome correlates of past sexual and physical trauma in 118 bipolar I disorder patients with a first episode of psychotic mania. Bipolar Disord 2010;12:244-252.

41. Kim SW, Kang HJ, Kim SY, Kim JM, Yoon JS, Jung SW, et al. Impact of childhood adversity on the course and suicidality of depressive disorders: the CRESCEND study. Depress Anxiety 2013;30:965-974.

42. Stange JP, Sylvia LG, da Silva Magalhães PV, Miklowitz DJ, Otto MW, Frank E, et al. Extreme attributions predict the course of bipolar depression: results from the STEP-BD randomized controlled trial of psychosocial treatment. J Clin Psychiatry 2013;74:249-255.

43. Stange JP, Sylvia LG, da Silva Magalhães PV, Miklowitz DJ, Otto MW, Frank E, et al. Extreme attributions predict suicidal ideation and suicide attempts in bipolar disorder: prospective data from STEP-BD. World Psychiatry 2014;13:95-96.

44. Kosel BW, Aweeka FT, Benowitz NL, Shade SB, Hilton JF, Lizak PS, et al. The effects of cannabinoids on the pharmacokinetics of indinavir and nelfinavir. AIDS 2002;16:543-550.

45. Stout SM, Cimino NM. Exogenous cannabinoids as substrates, inhibitors, and inducers of human drug metabolizing enzymes: a systematic review. Drug Metab Rev 2014;46:86-95.

46. Chetty M, Miller R, Moodley SV. Smoking and body weight influence 
the clearance of chlorpromazine. Eur J Clin Pharmacol 1994;46:523526 .

47. Kroon LA. Drug interactions with smoking. Am J Health Syst Pharm 2007;64:1917-1921.

48. Strakowski SM, Keck PE Jr, McElroy SL, West SA, Sax KW, Hawkins $\mathrm{JM}$, et al. Twelve-month outcome after a first hospitalization for affective psychosis. Arch Gen Psychiatry 1998;55:49-55.

49. Berk L, Hallam KT, Colom F, Vieta E, Hasty M, Macneil C, et al. Enhancing medication adherence in patients with bipolar disorder. Hum Psychopharmacol 2010;25:1-16.

50. González-Pinto A, Reed C, Novick D, Bertsch J, Haro JM. Assessment of medication adherence in a cohort of patients with bipolar disorder. Pharmacopsychiatry 2010;43:263-270.

51. de Leon J, Susce MT, Diaz FJ, Rendon DM, Velasquez DM. Variables associated with alcohol, drug, and daily smoking cessation in patients with severe mental illness. J Clin Psychiatry 2005;66:1447-1455.
52. Heffner JL, Del Bello MP, Anthenelli RM, Fleck DE, Adler CM, Strakowski SM. Cigarette smoking and its relationship to mood disorder symptoms and co-occurring alcohol and cannabis use disorders following first hospitalization for bipolar disorder. Bipolar Disord 2012;14:99108.

53. Peters EN, Schwartz RP, Wang S, O'Grady KE, Blanco C. Psychiatric, psychosocial, and physical health correlates of co-occurring cannabis use disorders and nicotine dependence. Drug Alcohol Depend 2014; 134:228-234.

54. Waxmonsky JA, ThomasMR, MiklowitzDJ, AllenMH, Wisniewski SR, Zhang $\mathrm{H}$, et al. Prevalence and correlates of tobacco use in bipolar disorder: data from the first 2000 participants in the Systematic Treatment Enhancement Program. Gen Hosp Psychiatry 2005;27:321-328.

55. Levin FR, Hennessy G. Bipolar disorder and substance abuse. Biol Psychiatry 2004;56:738-748. 